\title{
Determination of 3D PSFs from computed tomography reconstructed $x$-ray images of spherical objects and the effects of sphere radii
}

\author{
Normand Robert, James G. Mainprize, Cari Whyne
}

Version Post-print/accepted manuscript

Citation Robert, N., Mainprize, J.G. and Whyne, C. (2019), Determination of 3D

(published version) PSFs from computed tomography reconstructed x-ray images of spherical objects and the effects of sphere radii. Med. Phys., 46: 47924802. https://doi.org/10.1002/mp.13734

Publisher's Statement This is the peer reviewed version of the following article: Robert, N., Mainprize, J.G. and Whyne, C. (2019), Determination of 3D PSFs from computed tomography reconstructed x-ray images of spherical objects and the effects of sphere radii. Med. Phys., 46: 4792-4802. https://doi.org/10.1002/mp.13734, which has been published in final form at https://doi.org/10.1002/mp.13734. This article may be used for non-commercial purposes in accordance with Wiley Terms and Conditions for Use of Self-Archived Versions.

\section{How to cite TSpace items}

Always cite the published version, so the author(s) will receive recognition through services that track citation counts, e.g. Scopus. If you need to cite the page number of the author manuscript from TSpace because you cannot access the published version, then cite the TSpace version in addition to the published version using the permanent URI (handle) found on the record page.

This article was made openly accessible by $U$ of $T$ Faculty. Please tell us how this access benefits you. Your story matters. 


\title{
Determination of 3D PSFs from computed tomography
}

reconstructed x-ray images of spherical objects and the effects of sphere radii

\author{
Normand Robert
}

Sunnybrook Research Institute,

2075 Bayview Avenue, Toronto, Ontario, M4N 3M5, Canada

normand.robert@sri.utoronto.ca,

James G Mainprize

Sunnybrook Research Institute,

2075 Bayview Avenue, Toronto, Ontario, M4N 3M5, Canada, and

Cari Whyne

Sunnybrook Research Institute, 2075 Bayview Avenue, Toronto, ON, Canada

Department of Surgery, IBBME and IMS,

University of Toronto

(Dated: August 13, 2021) 


\begin{abstract}
Purpose: A method was developed to obtain three dimensional (3D) point spread functions (PSFs) of reconstructed x-ray volumetric images using spheres of known diameters. The algorithm consists of a sphere localization step using template matching applied to the entire volume. Richardson Lucy (RL) deconvolution is used atypically to determine the PSF from the reconstructed x-ray image and a model of the sphere. The resulting PSF is arbitrary i.e., there are no assumptions of separability or symmetry. Oversampling is not used, and sample spacing matches the image. The effect of sphere radius on PSF estimate reproducibility is investigated.

Methods: Phantoms were constructed by suspending 5 polytetrafluoroethylene (PTFE) spheres having known radii equal to 4.77, 7.95, 9.52, 12.68 and $19.53 \mathrm{~mm}$ in an agar solution. The phantom included a $25 \mu \mathrm{m}$ steel wire to calculate a line spread function (LSF). The phantom was imaged and reconstructed with a Medtronic surgical O-Arm 23 times and a Toshiba Aquilion One CT 20 times. A sharp reconstruction kernel exhibiting a non-monotonic PSF was used with the Toshiba CT. PSFs and LSFs were computed for all of the images and repeated estimates were used to compute mean and standard deviation values for every point of the PSFs and LSFs. The PSFs from spheres were converted to LSFs and compared to the wire LSF.

Results: The standard deviations of the PSF estimates exhibit a decreasing trend as the sphere radius is increased. The PSF from the smallest $4.77 \mathrm{~mm}$ sphere is the least reproducible. The normalized root mean square difference between the mean LSF derived from the $4.77 \mathrm{~mm}$ radius sphere and the mean wire LSF is $2.0 \%$ for the O-arm and $1.2 \%$ for the CT.

Conclusion: RL deconvolution provides a method to estimate generalized (no separability or other simplifying assumptions) 3D PSFs from spheres. X-ray noise in images acquired with typical clinical protocols cause noticeable variations in PSF estimates which can be mitigated by selecting larger spheres and combining PSF estimates from different images.




\section{INTRODUCTION}

Resolution and noise are principal determinants of image quality. The resolution of an image can be characterized by its point spread function (PSF) or equivalently via its spatial frequency counterpart known as the modulation transfer function (MTF). Noise is commonly characterized via the frequency space noise power spectrum (NPS). This paper investigates a method to calculate PSF estimates from three dimensional reconstructed xray images of spherical test objects and examines how sphere radius affects these estimates. Determination of PSFs or resolution surrogates such as the resolvable line-pair distance are used to evaluate and optimize the performance of new detector designs. Detective quantum in surgical planning. Pakdelet al.[1] have demonstrated how corrections reduce these biases.

Conceptually, the PSF can be obtained directly by imaging an infinitesimally small object. However, this is not practical as it results in a noisy estimate due to the small number of voxel values used to obtain the PSF. Thus, other methods have been developed whereby a measure For example, in x-ray imaging, a wire can provide a two dimensional measure of resolution known as the line spread function (LSF) [2]. Other approaches have been developed using a slit[3] and a thin sheet of foil[4]. Ohkubo et al. [5] used a cylinder and assumed $z$ separability to obtain a PSF in the transverse plane. Many of these approaches typically also involve response functions.

Several methods have been proposed to determine PSFs using spheres composed of materials having different attenuating properties than the surrounding medium[6-10]. Spheres are compact, require no alignment and can potentially provide PSF estimates in all directions. Chen et al.[6] used a $6 \mathrm{~mm}$ diameter polytetrafluoroethylene (PTFE) plastic sphere with the assumption of cartesian separability to obtain a 3D Gaussian PSF. PTFE has 
X-ray attenuation properties similar to bone. Thornton et al.[7] used PTFE spheres with diameters of $0.5,1.0$ and 1.5 inches to obtain surface spread functions (SPF)s over a conic section. The SPFs are smoothed using b-splines, differentiated and combined to yield PSFs. This approach requires the diameter of the sphere to be large compared to the width of the PSF. Steiding et al.[9] developed a quality assurance framework and phantom whereby a 12 mm diameter aluminum sphere is used to obtain a PSF using the aforementioned method described by Thornton et al.[7].

Lee et al.[10] described a method to determine the MTF in any arbitrary direction that arbitrary/generic PSF from spheres using RL deconvolution and investigate the performance of this approach for spheres of differing diameters. 


\section{THEORY}

\section{A. Sphere localization}

A robust strategy was developed to localize spheres in the presence of other image features without user interaction such as seeding of the sphere positions or limiting searches to volumes of interest. Our approach would allow for the spheres to be localized if used in conjunction with a clinical scan.

It is required that the spheres have known diameters and uniform compositions with x-ray attenuation properties that differ from the surrounding media in which they are suspended to create contrast. It is assumed that none of the voxel values for the various components in the phantom are known a-priori. Thus, the method is also applicable with voxel values having arbitrary scaling and offset i.e. values not expressed in Hounsfield units. These conditions are consistent with template matching using a three dimensional normalized cross-correlation (NCC)[13].

We define the target image $I(x, y, z)$ having $L_{I} \times M_{I} \times N_{I}$ voxels such that $x \in\left[0, L_{I}\right)$, $y \in\left[0, M_{I}\right) z \in\left[0, N_{I}\right)$. The template image $T(x, y, z)$ has $L_{T} \times M_{T} \times N_{T}$ voxels such that $x \in\left[0, L_{T}\right), y \in\left[0, M_{T}\right), z \in\left[0, N_{T}\right)$. The template contains a noise free model of the sphere and has a region of support just large enough to hold said sphere and is usually smaller than the image. The NCC of the target image and template image is written

$$
C(x, y, z)=\frac{\sum_{x^{\prime}, y^{\prime}, z^{\prime}} I\left(x^{\prime}, y^{\prime}, z^{\prime}\right)\left(T\left(x^{\prime}-x, y^{\prime}-y, z-z^{\prime}\right)-\bar{T}\right)}{\sqrt{\sigma_{I}^{2}(x, y, z) \sigma_{T}^{2}}},
$$

where

$$
\sigma_{I}^{2}(x, y, z)=\sum_{x^{\prime}=x}^{x+L_{T}-1} \sum_{y^{\prime}=y}^{y+M_{T}-1} \sum_{z^{\prime}=z}^{z+N_{T}-1}\left[I\left(x^{\prime}, y^{\prime}, z^{\prime}\right)-\overline{I(x, y, z)}\right]^{2}
$$

$$
\begin{gathered}
\sigma_{T}^{2}=\sum_{x^{\prime}, y^{\prime}, z^{\prime}}\left[T\left(x^{\prime}, y^{\prime}, z^{\prime}\right)-\bar{T}\right]^{2}, \\
\overline{I(x, y, z)}=\sum_{x^{\prime}=x}^{x+L_{T}-1} \sum_{y^{\prime}=y}^{y+M_{T}-1} \sum_{z^{\prime}=z}^{z+N_{T}-1} I\left(x^{\prime}, y^{\prime}, z^{\prime}\right) /\left(L_{T} M_{T} N_{T}\right),
\end{gathered}
$$

and

$$
\bar{T}=\sum_{x^{\prime}, y^{\prime}, z^{\prime}} T\left(x^{\prime}, y^{\prime}, z^{\prime}\right) /\left(L_{T} M_{T} N_{T}\right)
$$


The $N C C$ is a measure of how well the template shifted by $(x, y, z)$ resembles the target image whereby $C(x, y, z,) \in[-1 ., 1$.$] . Due to rotational symmetry of the template which$ contains a sphere, there is no need to repeat the NCC calculation for different rotations of the template. If the target image is $I(x, y, z)=a T\left(x-x_{0}, y-y_{0}, z-z_{0}\right)+b$, with $a \neq 0$ then the NCC operation between $I(x, y, z)$ and $T(x, y, z)$ would yield a maximum for $C$ at $\left(x_{0}, y_{0}, z_{0}\right)$ such that $C\left(x_{0}, y_{0}, z_{0}\right)=1.0$ indicating a perfect fit independent of $a$ and $b$ which is the behavior we want. The NCC like other template matching methods is computationally intensive but it can be speed-ed up using FFTs and sum tables also known as prefix sums[14, 15]. Localization is used to define a VOI encompassing the sphere. The VOI for each sphere is padded to allow for sampling of the effects of the PSF tail but limited in extent to reduce noise[16].

\section{B. Sphere Model}

A template voxel volume is created to hold a sphere model at its center. The voxel count is made sufficient to support the sphere (no truncation). The model of the sphere is calculated by giving voxels within the radius of the sphere a value of 1 and points outside a value of 0 given by

$$
S\left(x, y, z, x_{0}, y_{0}, z_{0}, R\right)= \begin{cases}1 \text { if } & \left(x-x_{0}\right)^{2}+ \\ & \left(y-y_{0}\right)^{2}+ \\ & \left(z-z_{0}\right)^{2}<R^{2} \\ 0 & \text { otherwise }\end{cases}
$$

The sphere can be generated with voxel spacings $Q$ times smaller in all directions than the voxel spacing used for $I(x, y, z)$. The high resolution sphere model is subsequently downsampled to exhibit the same voxel spacing as $I(x, y, z)$ producing a linear partial volume effect. The sphere model is used to locate spheres and also to determine PSFs. When determining the PSF, we set $Q=1$ so the model has hard edges because PSF determination only needs an approximate seed position for the sphere. Furthermore, sphere localization may be done on a downsampled image to speed up calculations. The PSF centers are identified when data from different scans are combined in a subsequent step described in Sec. II E. 


\section{PSF determination}

Richardson and Lucy deconvolution [11, 12] involves convolution operations which can be computed via fast Fourier transforms and parallelized. Many computing environments

160

165 geometrical scaling factor, $M$, for the sphere model radius to eliminate said errors. This 
factor is determined by repeating the PSF calculation and sphere position are varied in a loop driven by simplex optimization whereby RMS differences between the image of the sphere and sphere model are minimized. Linear regression is performed to match image and sphere model voxel values at each loop step by rescaling the model such that $o^{\prime}(x, y, z)=$ $o(x, y, z) m+b$

\section{Comparison to LSF via wire method}

The phantom contains a wire allowing a determination the line spread function (LSF) in written as

$$
P S F(x, y, z)=P S F_{x y}(x, y) P S F_{z}(z) .
$$

Substituting Eq. 9 into Eq. 7 yields

$$
L S F(x, y)=P S F_{x y}(x, y) \int_{-\infty}^{\infty} P S F_{z}(z) d z
$$


from the prescribed center indices is re-computed and interpolation is done from the original data as before but with the addition of said residual distance. This latter step is repeated until the COM estimate is within a small voxel spacing fraction from the prescribed voxel indices but with the interpolation always done from the original data with increasingly 

with a peak at the prescribed indices. This approach is also used for LSF estimates.

\section{EXPERIMENTAL METHODS}

\section{A. Sphere and Wire Phantom}

Fisher Scientific BD Difco Agar was added to deionized degassed water (1.5\% by weight) tion kernel, 0.828 pitch factor, TCOT reconstruction algorithm, AIDR 3D STD filter, voxel 
spacing of $(0.421,0.421,0.30) \mathrm{mm}, 120 \mathrm{kVP}, 0.9 \mathrm{~mm}$ focal spot size, $500 \mathrm{~ms}$ exposure, 250 $\mathrm{mA}$ tube current and a CTDI of $14 \mathrm{mGy}$. In this manuscript, image directions 1 and 2 refer the transverse imaging plane. The x-ray source and detector rotation axis corresponds to direction 3 .

\section{Image quality}

We record several measures of image fidelity/quality due to their effects on PSF accuracy and reproducibility. The spatial mean and standard deviation of voxels value are computed for each scan. The RMS variations in these statistics are measures of signal reproducibility. There are many possible causes for variations in voxel values including fluctuations in supply voltage to the imaging unit, heating of components affecting their behavior as well as changes in detector gain. By the properties of convolutions, a change in signal scaling or offset will respectively affect the PSF value scaling and offset.

The reproducibility in sphere positions is estimated by computing standard deviations of the COM of the spheres for the repeated scans. Mechanical play can cause variations in source-detector orbits relative to the object to be imaged e.g. couch motion in a spiral scan. These variations cause shifts in the positions of PSF and LSF estimates. Uncontrolled variations in sphere positions would increase the standard deviation of the PSFs and LSFs unless these variations are estimated and corrected.

\section{PSF / LSF generation}

The images are read using Matlab (MathWorks, Natick, MA, USA) DICOM utilities wrapped within DICOM classes we have developed. The three dimensional voxel spacing is obtained from the DICOM files so that non isotropic spacing is taken into account i.e. sphere models and PSF estimates have the correct scaling in every direction. These data are passed to our PSF sphere class as well as another class implementing the wire LSF method. The 5 spheres are found and the mean voxel values and standard deviation within $2.5 \mathrm{~mm}$ radius volumes of interests (VOI)s centered on each sphere are obtained to investigate how changing sphere radius affects image noise. For each sphere, the sphere model and VOI encompassing the sphere are used to obtain a PSF estimate. The VOIs containing the 
sphere image volumes include 5.4 to $6.4 \mathrm{~mm}$ of padding on all sides to allow for sampling for the PSF "tail". The padding was constrained by the separation of the spheres placed along a line so their projections do not overlap and such that the entire phantom can be imaged with the O-arm which has no spiral acquisition option. The sphere model is generated with a fourfold increase in voxel density and subsequently downsampled to create partial volume effects.

PSFs from spheres of different diameters and in different locations exhibit some differences in scaling and offset due to local changes in the images. Given $n$ PSF functions obtained under different conditions (e.g. different sphere radii), we transform the PSF values for the $i$ th imaging condition where $i \in(2, n)$ such that $P S F_{i}^{\prime}(x, y, z)=P S F_{i}(x, y, z) m_{i}+b_{i}$ where $m_{i}$ and $b_{i}$ are calculated via linear regression with the first PSF. Linear regression distributes difference between PSF evenly over their domains.

There are different preferred conventions for normalizing PSFs including a central peak value of one, normalization by the volume under the PSF, which can be done in voxel units or spatial units e.g. mm. We chose the latter, such that

$$
P S F^{\prime}(x, y, z)=P S F(x, y, z) /\left[\sum P S F(x, y, z) \Delta x \Delta y \Delta z\right]
$$

where $\Delta x, \Delta y, \Delta z$ are the voxel spacings in the $x, y, z$ directions yielding 3D PSFs in $\mathrm{mm}^{-3}$ and similarly, 2D LSFs in $\mathrm{mm}^{-2}$. The "raw" PSFs from our approach (sphere model $\rightarrow$ image) have values ranging from approximately zero in the periphery to hundreds at the centre, a consequence of the sphere model with values ranging between zero and one, the phantom materials, which are determinants of voxel value ranges in Hounsfield Unit (HU) for CT and in arbitrary units (AU) for the O-arm. PSFs and LSFs should be adjusted to have values that tend to zero in the periphery otherwise normalization may not yield the expected results. In our process, whereby several results are shown together, we calculate the normalization factor (volume under the curve) from the first PSF or LSF in the sequence and apply said factor to the first and remaining linearly regressed PSFs identically. Therefore, the relative closeness between the PSFs is unaffected by normalization and is still governed by linear regression. 


\section{E. PSF versus RL iterations} boundary indicate that the PSF estimate cannot account for the x-ray data. the estimated PSF will be significantly different from the true PSF. RL can alternatively be susceptible to producing estimates with high frequency noise with some data sets when too many iterations are used[19]. PSFs are calculated using 30, 60, 800, 1600 RL iterations. The PSF computation time on a legacy Intel Core i7 CPU $9302.80 \mathrm{GHz}$ circa 2010 for 800 iterations with offset correction for the small sphere is $90 \mathrm{~s}$.

\section{F. PSF accuracy surrogate}

In the absence of an independent PSF estimate that can be treated as truth, an indicator of accuracy of our approach is computed by convolving our PSF estimate with the object model and subtracting the result from the x-ray volume image to yield a difference volume as was done by Ohkubo et al. [5]. Residuals in a difference volume particularly at the sphere

\section{G. LSF comparison}

LSFs were computed by spatial integration of PSFs along direction 3 as written in Eq. 8 matching the direction of the wire. LSFs derived from sphere PSFs have different amplitudes

RL requires the prescription of the number of iterations. If too few iterations are used, and level than the wire LSFs. The wire LSFs also exhibits higher sampling densities. Sample point harmonization via interpolation of the sphere LSF data facilitates alignment of central features from both methods which is useful for plotting. It also allows linear regression of the sphere LSF with a 2 parameter voxel value transformation model of the form $L S F^{\prime}(x, y, z)=$ $\operatorname{LSF}(x, y, z) m+b$ so these data match the wire LSFs. A difference image is calculated between the modified LSF from the $4.77 \mathrm{~mm}$ sphere and wire LSF as well RMS differences. 


\section{RESULTS AND DISCUSSION}

\section{A. Image quality and reproducibility}

The RMS variations in voxel value mean and standard deviation over the entire images between repeated scans were 1.5 and 1.6 voxel units respectively for the O-arm and $0.11 \mathrm{HU}$

and 0.11 HU respectively for the CT scanner. These RMS variations are small relative to the voxel value range and x-ray noise and are thus not expected to affect PSF reproducibility. The O-arm voxel values are approximately 2.7 times greater than the Hounsfield units CT values for PTFE which has similar attenuation property as bone.

Table I shows the spatial means and standard deviations of voxel values for a spherical volumes of interest (VOI) having a $2.5 \mathrm{~mm}$ radius centered on each PTFE sphere. The number of voxels in the VOIs vary slightly depending on the fractional location of the sphere center in voxel units. For each sphere diameter, VOIs were extracted from each of the images allowing the inclusion of reproducibility estimates for the mean and standard deviation i.e. " \pm " shown in Table I. The standard deviations of the voxel values are a larger fraction of the mean values for the CT than the O-arm with the imaging protocols we have chosen. The VOI standard deviation, an estimate of image noise, exhibits little change as a function of sphere diameter. Although larger attenuation spheres absorb more x rays, the loss of $\mathrm{x}$-ray photons has little effect on the noise of the reconstructed x-ray image. These results suggest that increasing the sphere diameter would be advantageous due to an increase in sphere boundary surface area with no associated changes in image noise.

Table II provides estimates of COM standard deviations along the 3 principal image axes as a measure of scan position reproducibility for different radii and scanners. These estimates are affected by deviations from the prescribed scanning geometry as well as x-ray noise. The deviations are larger for the CT data. In most instances, the deviations are small fraction of the voxel sizes.

\section{B. PSF and RL iterations}

Figure 2 shows PSF estimates for the O-arm along one principal axis

of the x-ray image for a $12.68 \mathrm{~mm}$ radius sphere computed with 30, 60, 800 and $1600 \mathrm{RL}$ iterations. The numerical values of the PSF have not been normalized and the large values 
$(\gg 1)$ are due to differences in scaling between the sphere model and the x-ray image. Each point on the PSFs is the mean value from 23 estimates computed from the 23 images. The error bars are the standard deviations of the repeated estimates (not the standard error of the mean). The PSF values stabilize around 400-800 iterations and additional iterations have little noticeable effect.

Figure 3 shows differences

between the sphere model convolved with the PSF and the O-arm reconstruction for the $12.68 \mathrm{~mm}$ sphere. A slice bisecting the sphere was chosen to illustrate these differences. The aspect ratio of the images have been corrected to compensate for non isotropic voxel spacing resulting in non square voxels. The difference images are computed for 30, 60, 800 and 1600 RL iterations. The differences are reduced as the number of iterations is increased and little change is observed between 800 and 1600 iterations.

\section{Image spatial scaling from sphere PSFs}

The image scaling estimates obtained from the $12.68 \mathrm{~mm}$ radius sphere were $1.0013 \pm 0.00020$ for the O-arm and $1.0008 \pm 0.00024$ for the CT. These values are closer to unity than we expected indicating a well prescribed gantry geometry and/or a careful system calibration. We also confirmed that PSFs computed with and without spatial corrections were nearly identical.

\section{PSF center of mass variation}

Table III shows variations in COM of the PSFs along the three principal image directions for 3 of the 5 radii. The superscripts ${ }^{c}$ indicates the correction as detailed in Sec. II E whereby image data is shifted via interpolation according to the COMs of the spheres prior to calculating measures of resolution and combining them. For the O-arm data all reproducibility estimates $(5 \times 3)$ except for three are smaller with motion correction. O-arm results are mixed which we suspect is due to the greater x-ray noise reducing sphere COM reproducibility. 


\section{E. PSF and sphere diameter}

Figure 4 shows the variability in the 23 PSF estimates obtained from repeated image O-arm acquisitions.

Figure 5 shows O-arm and CT PSF estimates along the 3 principal axes of the

x-ray image for the 4.77, 7.95, 9.52, 12.68 and $19.05 \mathrm{~mm}$ radius spheres. Linear regression was performed to match the PSF scaling and offset. The O-arm PSFs exhibit the expected monotonic decrease within a few pixels from their centers associated with a "soft" reconstruction kernel. The CT PSFs exhibits valleys on both sides of the peak associated with a sharp reconstruction kernel which required adding an offset to the convolved images and compensating for this offset so that a zero crossing PSF is calculated correctly (Sec. II C). The CT PSFs are in general agreement but the valleys are attenuated at larger diameter. The valley should mostly be radii invariant as they are governed by the reconstruction kernel and we can see edge enhancement for all sphere diameters. We suspect that in this simple two material image, non-linear effect such as beam hardening, are causing systematic errors.

The standard deviations of PSF values were calculated over their central regions encompassing $9 \times 9 \times 9$ voxels. The said deviations for sphere radii of 4.77, 7.95, 9.52 and $19.05 \mathrm{~mm}$ were respectively $0.80,0.12,0.07,0.01$ for the $\mathrm{O}$-arm and $0.24,0.06,0.02,0.01$ for the CT. This behavior is expected given that the image noise mostly independent of sphere size for spheres ranging from 4.77 to $19.05 \mathrm{~mm}$ and the increase in diameter provides a larger surface area boundary from which to estimate the PSF. While this measure may seem arbitrary due to the definition of the domain over which it is calculated, this measure which is applied consistently for the different PSF demonstrates how PSF noise relates to sphere diameter.

Figure 6 shows differences between the

sphere model convolved with the PSF and the O-arm reconstruction as explained in Section IV B for Figure 3. In this instance, the number of RL iterations is fixed at 800 and the sphere radii are varied. We have identified three possible mechanisms causing spherical artifacts in the difference images to become more noticeable with increasing diameter. Firstly, we know from contrast detail phantoms that features become more noticeable in noisy images with an increase in size. Secondly, larger spheres exacerbate non-linearities in the imaging system due to the polychromatic nature of the x-ray beam. Thirdly, we know that PSFs in cone beam geometries vary spatially and the use of a larger spheres results in an 
averaged PSF which may not be applicable at any given location. A different experimental protocol and phantom design is needed to explore this effect.

The spheres as shown in Fig.1 define an axis that is almost parallel to the rotation axis of

\section{SUMMARY AND CONCLUSIONS}

Richardson Lucy deconvolution can be used to estimate three dimensional PSFs from spheres of known diameters. RL implementations are ubiquitous making this approach easy to implement. We verified whether repeated scans were reproducible in terms of positional 
variations, spatial scaling, signal values and we developed corrections to prevent these variations from affecting PSF reproducibility and accuracy. Our design incorporating many images allowed the calculation of the mean and standard deviations of all points of the PSFs and LSFs. Sphere COM corrections improved PSF reproducibility for o-arm data but not for the CT data. COM estimates maybe more robust if confined to a shell encompassing the sphere boundary. The standard deviations of the PSF estimates exhibit a decreasing trend as the sphere radius was increased suggesting that the method requires a minimum sphere radius to yield the desired threshold of reproducibility. In the case of the O-arm scan, the PSFs are similar for all diameters. For the CT scan, the PSF shapes are similar but with a reduction in the valleys seen on both sides of the peak for larger radii. Our results suggest that there exists a trade-off between reproducibility and accuracy when the radius is increased as demonstrated by the structured noise of Fig. 6 indicating that the transfer function can not account for the image data. The experimental design was focused on obtaining measures of PSF reproducibility but sphere derived LSF measurements were compared to a wire LSF obtained in physical proximity. PSF are mostly $z$ separable and thus LSF agreement in this instance implies some agreement of the PSF in the $x y$ direction.

Noise has been identified as a challenge in all methods to determine PSFs and various regularization strategies have been proposed. Our approach uses an extended object relative to the voxel size and all voxels within a VOI to obtain the PSF reducing sensitivity to x-ray noise. Our approach also shows how PSF reproducibility can be improved by combining corrected estimates from different scans. At the same time, the absence of assumptions regarding the PSF such as separability or even-ess increase sensitivity to noise. Our approach does not provide oversampled or pre-sampled estimates of the PSF although this information can be extracted from sphere phantoms. Additional experiments are required to determine the level of PSF reproducibility required for our application involving the restoration of signal values and thicknesses of thin bones.

\section{ACKNOWLEDGMENTS}

We wish to acknowledge the financial support of the Sunnybrook Holland Musculoskeletal Program and the National Science and Engineering Council of Canada NSERC (239206). 


\section{CONFLICT OF INTEREST DISCLOSURE}

The authors have no relevant conflicts of interest to disclose.

[1] A. Pakdel, J. G. Mainprize, N. Robert, J. Fialkov, and C. M. Whyne. "Model-based PSF and MTF estimation and validation from skeletal clinical CT images", Medical physics 41, 011906 (2014).

[2] E. L. Nickoloff. "Measurement of the PSF for a CT scanner: appropriate wire diameter and pixel size", Physics in medicine and biology 33, 149 (1988).

[3] H. Fujita, D.-Y. Tsai, T. Itoh, J. Morishita, K. Ueda, A. Ohtsuka, et al. "A simple method for determining the modulation transfer function in digital radiography", Medical Imaging, IEEE Transactions on 11, 34-39 (1992).

[4] J. M. Boone. "Determination of the presampled MTF in computed tomography", Medical Physics 28, 356-360 (2001).

[5] M. Ohkubo, S. Wada, T. Matsumoto, and K. Nishizawa. "An effective method to verify line and point spread functions measured in computed tomography", Medical Physics 33, 2757-2764 (2006).

[6] Z. Chen and R. Ning. "Three-dimensional point spread function measurement of cone-beam computed tomography system by iterative edge-blurring algorithm", Physics in medicine and biology 49, 1865 (2004).

[7] M. M. Thornton. "Measurement of the spatial resolution of a clinical volumetric computed tomography scanner using a sphere phantom", Proceedings of SPIE 6142, 61421Z-61421Z-10 (2006).

[8] J. Baek and N. J. Pelc. "Use of sphere phantoms to measure the 3D MTF of FDK reconstructions", Physics 7961, 79610D-79610D-6 (2011).

[9] C. Steiding, D. Kolditz, and W. A. Kalender. "A quality assurance framework for the fully automated and objective evaluation of image quality in cone-beam computed tomography.", Medical physics 41, 031901 (2014).

[10] C. Lee and J. Baek. "A New Method to Measure Directional Modulation Transfer Function Using Sphere Phantoms in a Cone Beam Computed Tomography System”, IEEE Transactions 
on Medical Imaging 34, 902-910 (2015).

[11] W. H. Richardson. "Bayesian-based iterative method of image restoration*", J. Opt. Soc. Am. 62, 55-59 (1972).

[18] D. S. C. Biggs and M. Andrews. "Acceleration of iterative image restoration algorithms", Applied Optics 36, 1766 (1997).

[19] M. K. Khan, S. Morigi, L. Reichel, and F. Sgallari. "Iterative methods of Richardson-Lucytype for image deblurring", Numerical Mathematics 6, 262-275 (2013). 


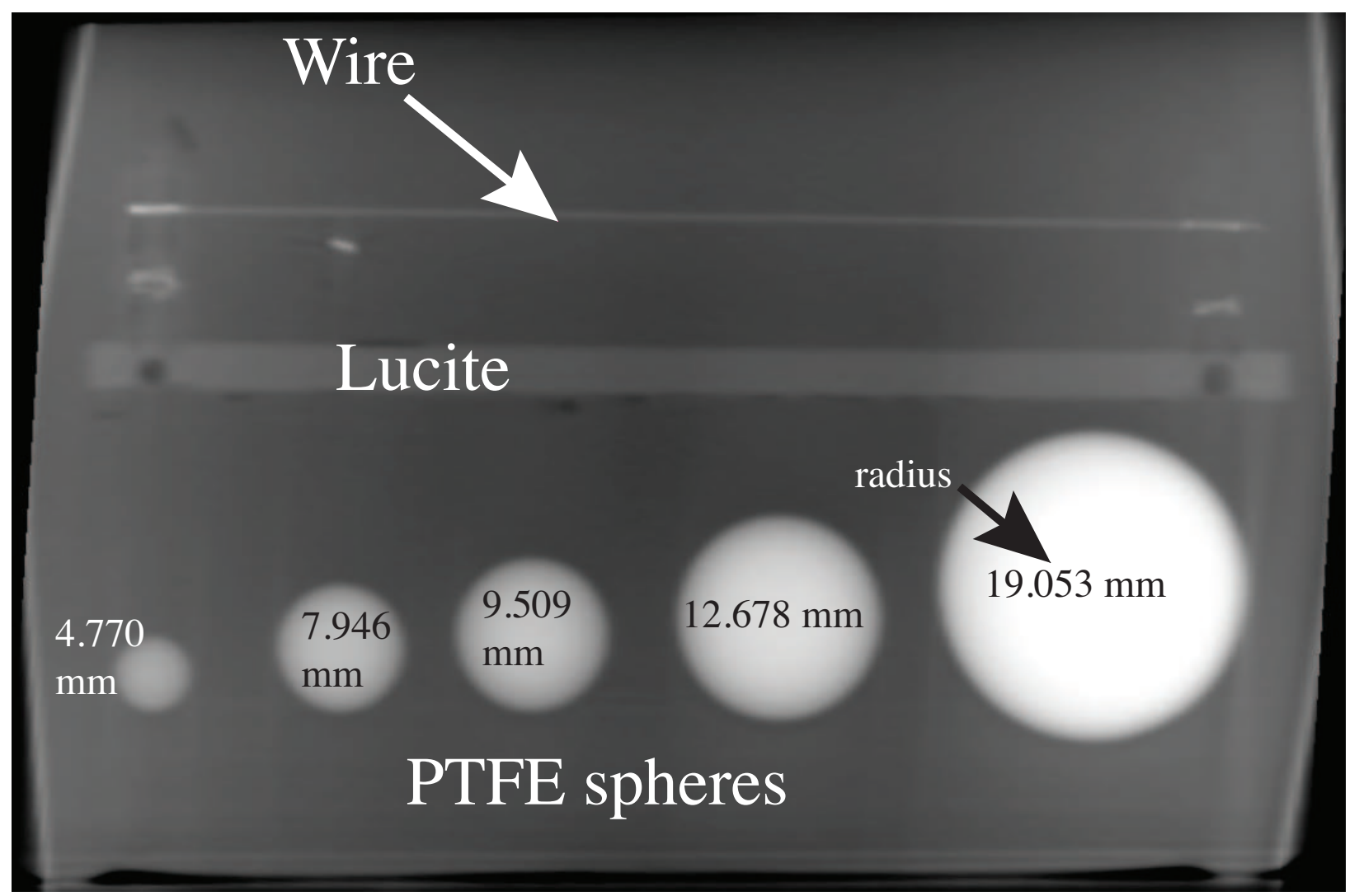

FIG. 1. A projection of a 3D x-ray image of an agar phantom containing PTFE spheres of varying diameters and a steel wire.

\begin{tabular}{|c|c|c|c|c|}
\hline Scanner & Radius (mm) & VOI Mean & VOI Std Dev & \# Voxels \\
\hline $\mathrm{O}$-arm & 4.77 & $2691 \pm 5.2$ & $24.1 \pm 1.1$ & $460 \times 23$ \\
\hline O-arm & 7.95 & $2561 \pm 3.9$ & $19.0 \pm 1.3$ & $466 \times 23$ \\
\hline O-arm & 9.52 & $2442 \pm 3.0$ & $19.7 \pm 1.1$ & $462 \times 23$ \\
\hline O-arm & 12.68 & $2430 \pm 3.5$ & $19.5 \pm 0.8$ & $465 \times 23$ \\
\hline O-arm & 19.05 & $2610 \pm 4.8$ & $23.8 \pm 1.1$ & $464 \times 23$ \\
\hline $\mathrm{CT}$ & 4.77 & $920 \pm 1.0$ & $19.3 \pm 1.1$ & $1217 \times 20$ \\
\hline $\mathrm{CT}$ & 19.05 & $940 \pm 1.0$ & $21.2 \pm 1.2$ & $1223 \times 20$ \\
\hline
\end{tabular}

TABLE I. The mean and standard deviation of voxel values within polytetrafluoroethylene spheres of different radii imaged using the Medtronic O-arm and Toshiba Aquilion One 320 CT. Errors in these estimates $( \pm)$ are available because each estimate was obtained from 23 image for the O-arm and 20 images for the CT. 


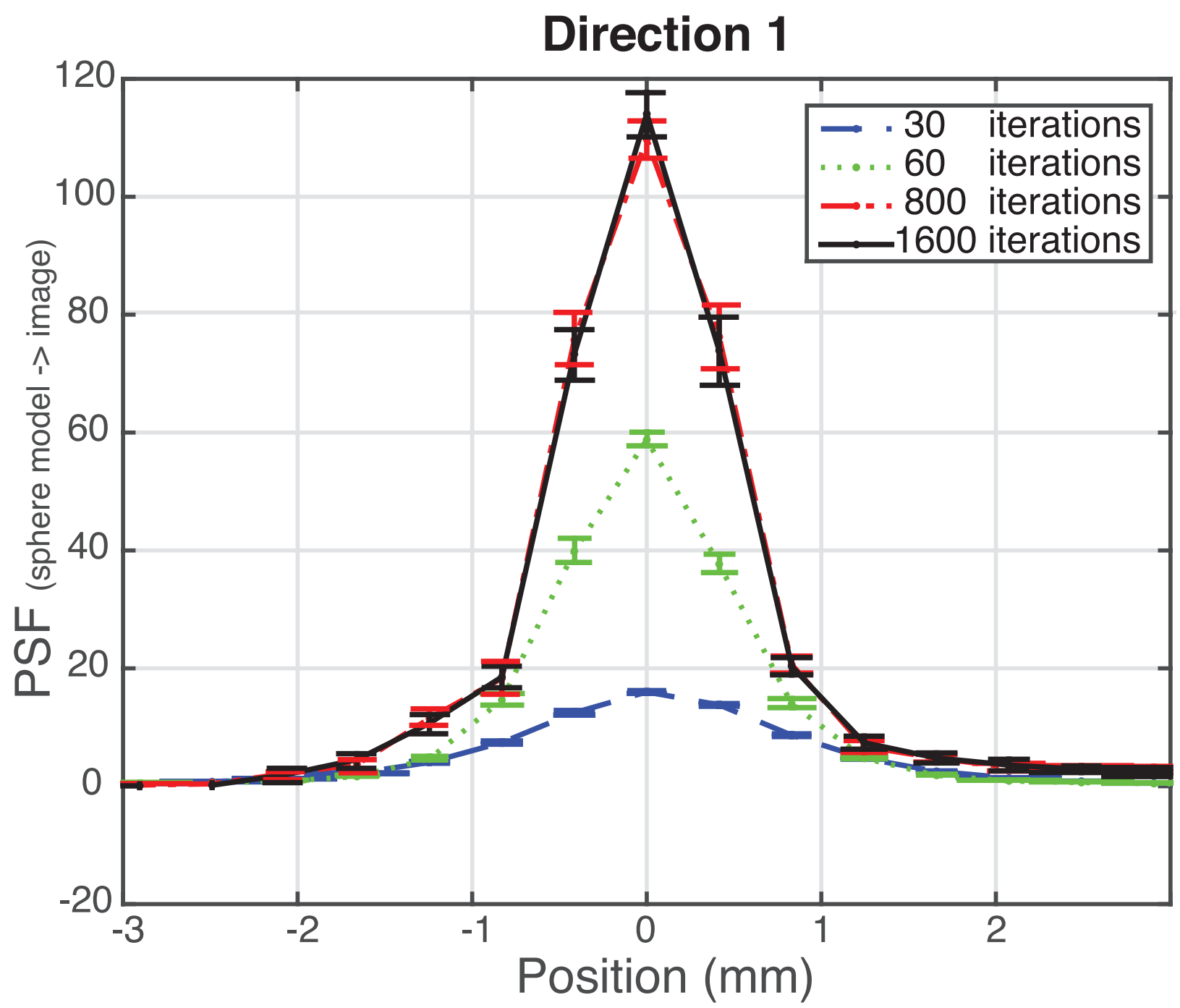

FIG. 2. Mean PSF estimates and standard deviations via error bars calculated along one principal axis of the O-arm images for a $12.68 \mathrm{~mm}$ radius sphere computed with 30, 60, 800 and $1600 \mathrm{RL}$ iterations. 


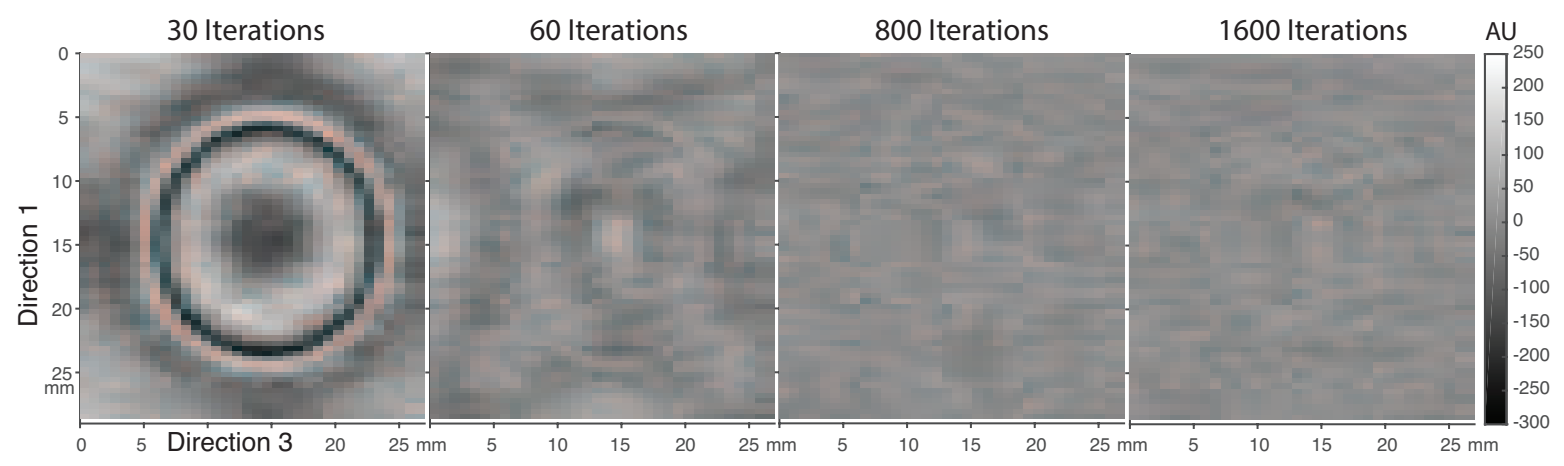

FIG. 3. Differences between the sphere model convolved with the PSF and the O-arm reconstruction for the $12.68 \mathrm{~mm}$ sphere with varying number of Richardson Lucy iterations. All images are windowed/leveled to same voxel value range as indicated by the color bar.

\begin{tabular}{ccccc}
\hline \hline Scanner & Radius $(\mathrm{mm})$ & $\mathrm{t}$ & \\
\hline O-arm & 4.77 & $(0.0027 / 0.0022$, & $0.0024 / 0.0010$, & $0.0032 / 0.0013)$ \\
O-arm & 9.52 & $(0.0034 / 0.0028$, & $0.0074 / 0.0031$, & $0.0031 / 0.0013)$ \\
O-arm & 19.05 & $(0.0062 / 0.0052$, & $0.0113 / 0.0047$, & $0.0048 / 0.0020)$ \\
CT & 4.77 & $(0.1189 / 0.0357$, & $0.0933 / 0.0393$, & $0.1242 / 0.0523)$ \\
CT & 9.52 & $(0.0585 / 0.0175$, & $0.1381 / 0.0581$, & $0.1778 / 0.0748)$ \\
CT & 19.05 & $(0.0856 / 0.0257$, & $0.0367 / 0.0155$, & $0.0492 / 0.0207)$ \\
\hline \hline
\end{tabular}

TABLE II. Standard deviations in center of mass sphere positions for varying radii (3 of 5 are shown ) expressed in voxel units and mm along the 3 principal image axes for 23 image from the O-arm and 20 images from the CT 


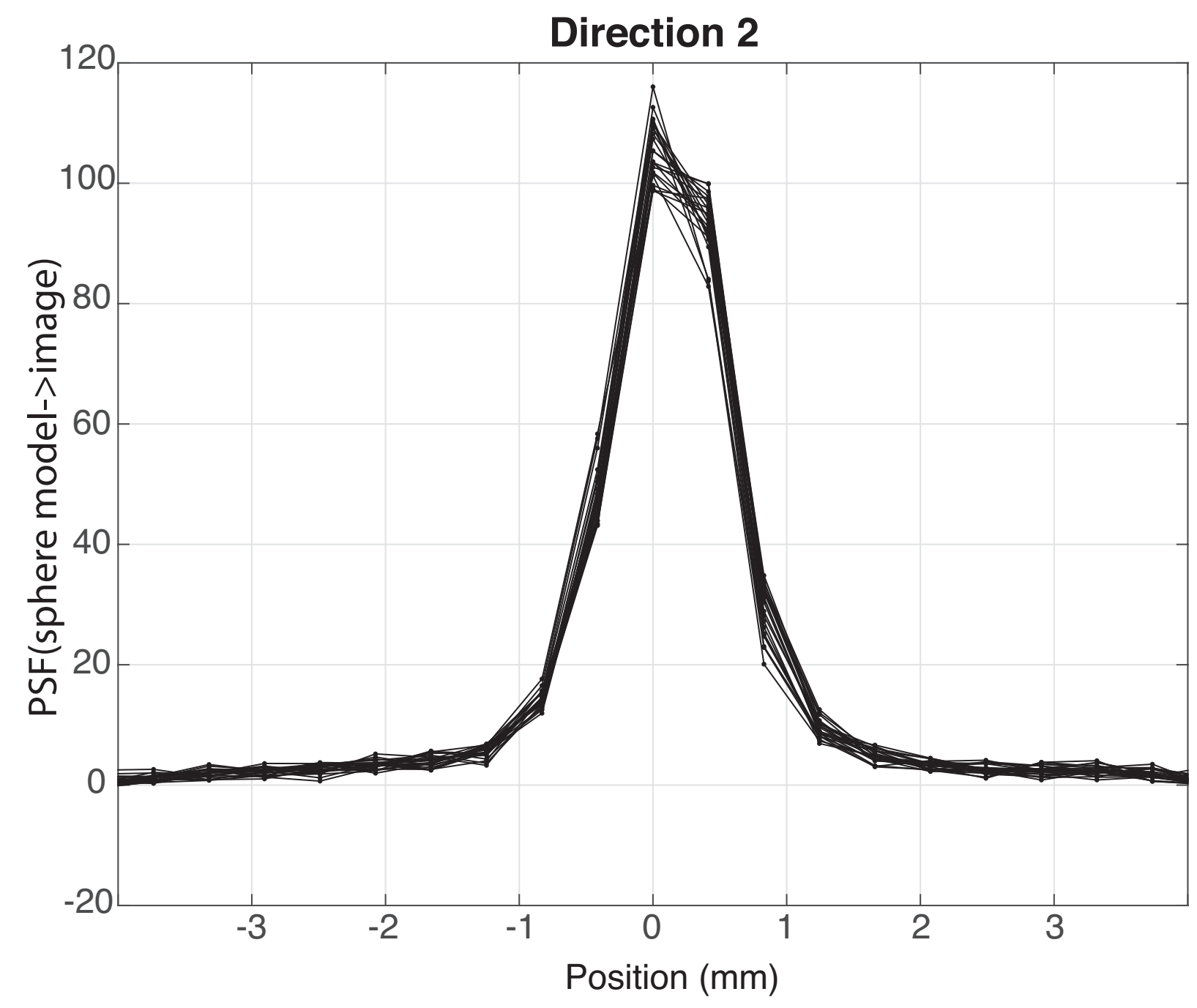

FIG. 4. PSF estimates from the 23 repeated O-arm images calculated along the second principal image axis for a $12.68 \mathrm{~mm}$ radius sphere. 


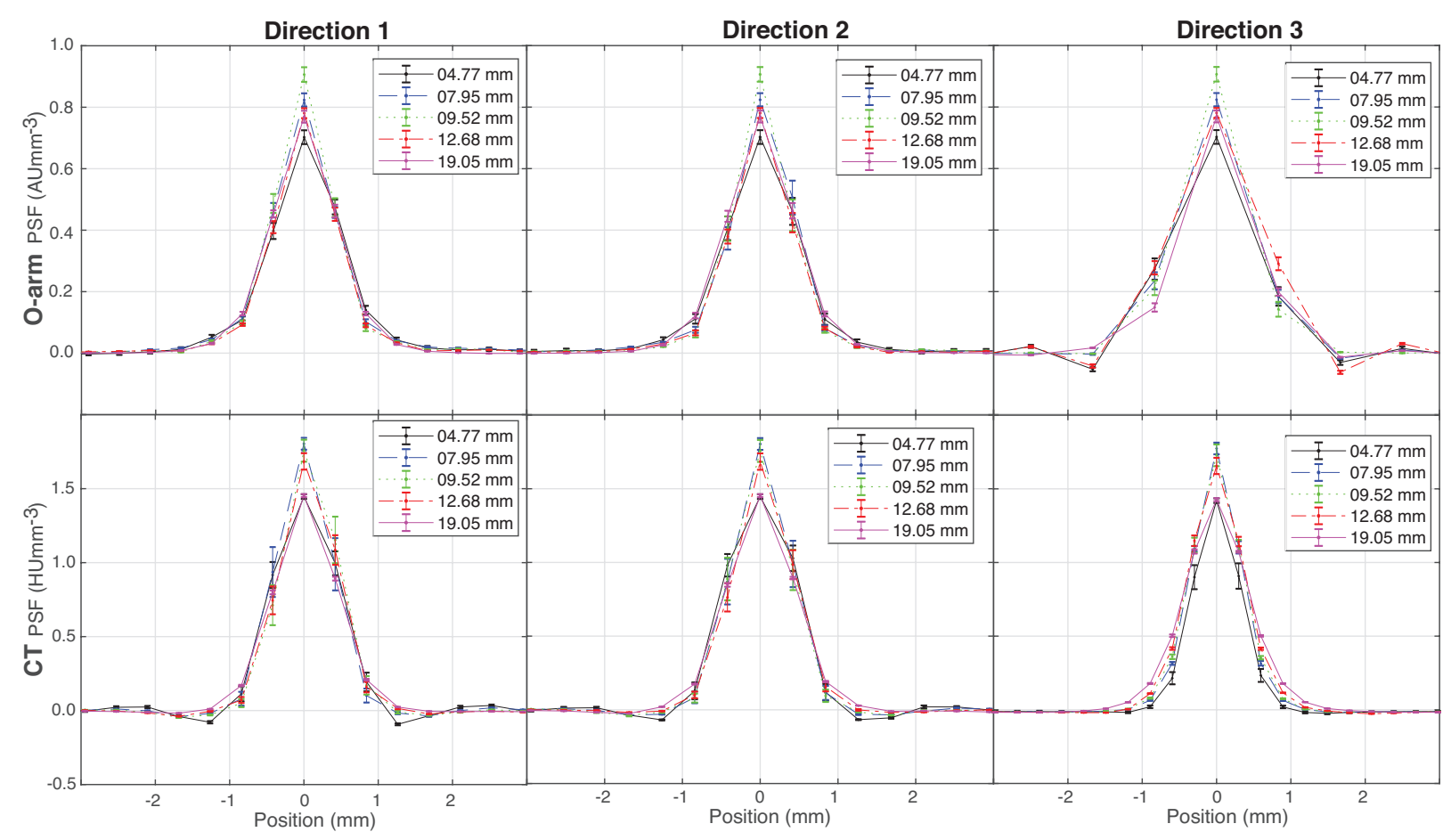

FIG. 5. Mean PSF estimates and standard deviations via error bars calculated along the 3 principal axes for spheres of different radii for images acquired with the O-arm (top row) and CT (bottom row)



FIG. 6. Differences between the sphere model convolved with the PSF and the x-ray reconstruction for 800 Richardson Lucy iterations and varying radii $r$. The first row shows results obtained with the O-arm and the second row show CT results. The VOI sizes increase to encompass the spheres as their radii increase resulting in smaller apparent voxel sizes. 


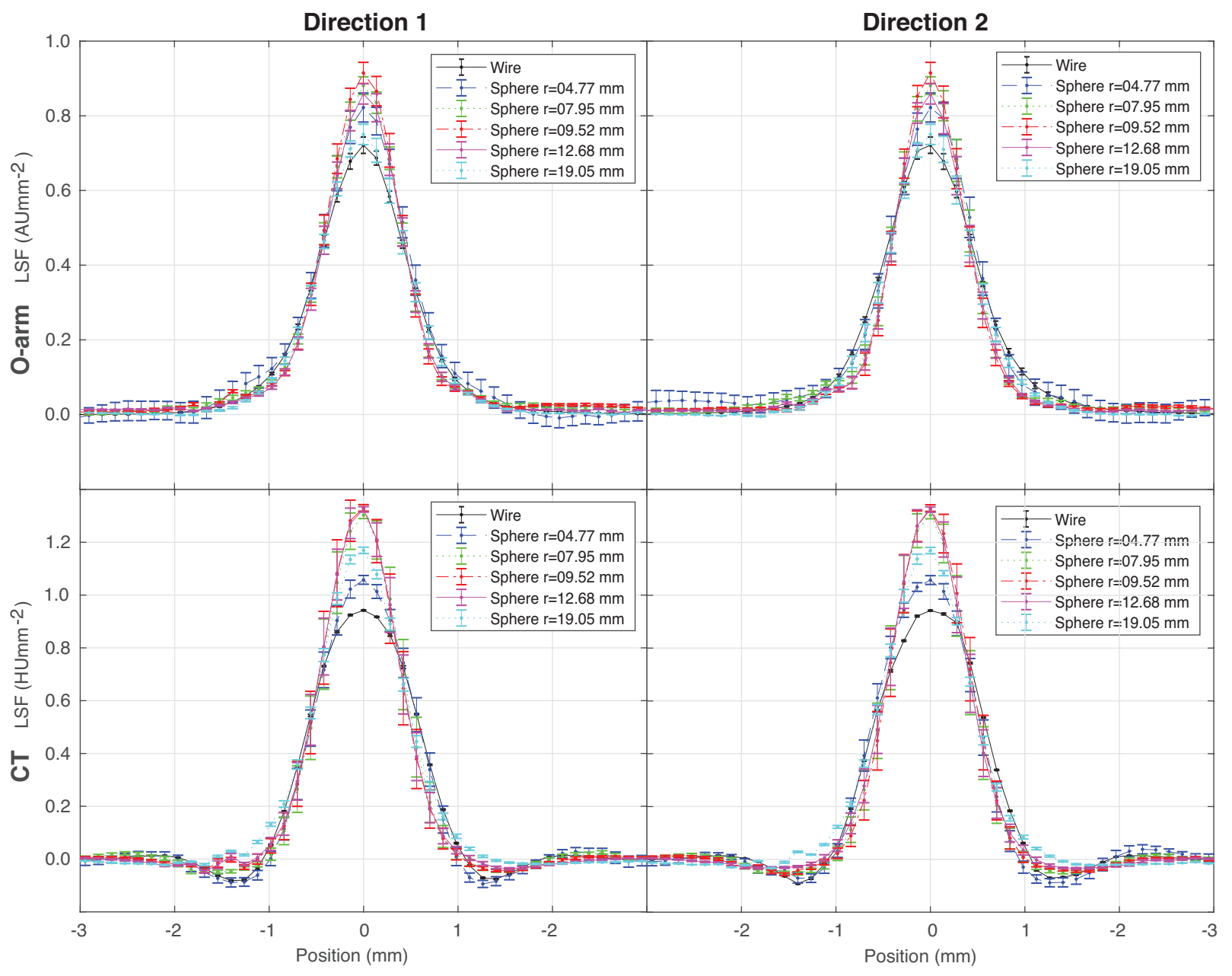

FIG. 7. Mean line spread function estimates and standard deviations via error bars calculated from 5 spheres of different radii and a wire for images acquired with the O-arm (top row) and CT (bottom row) 

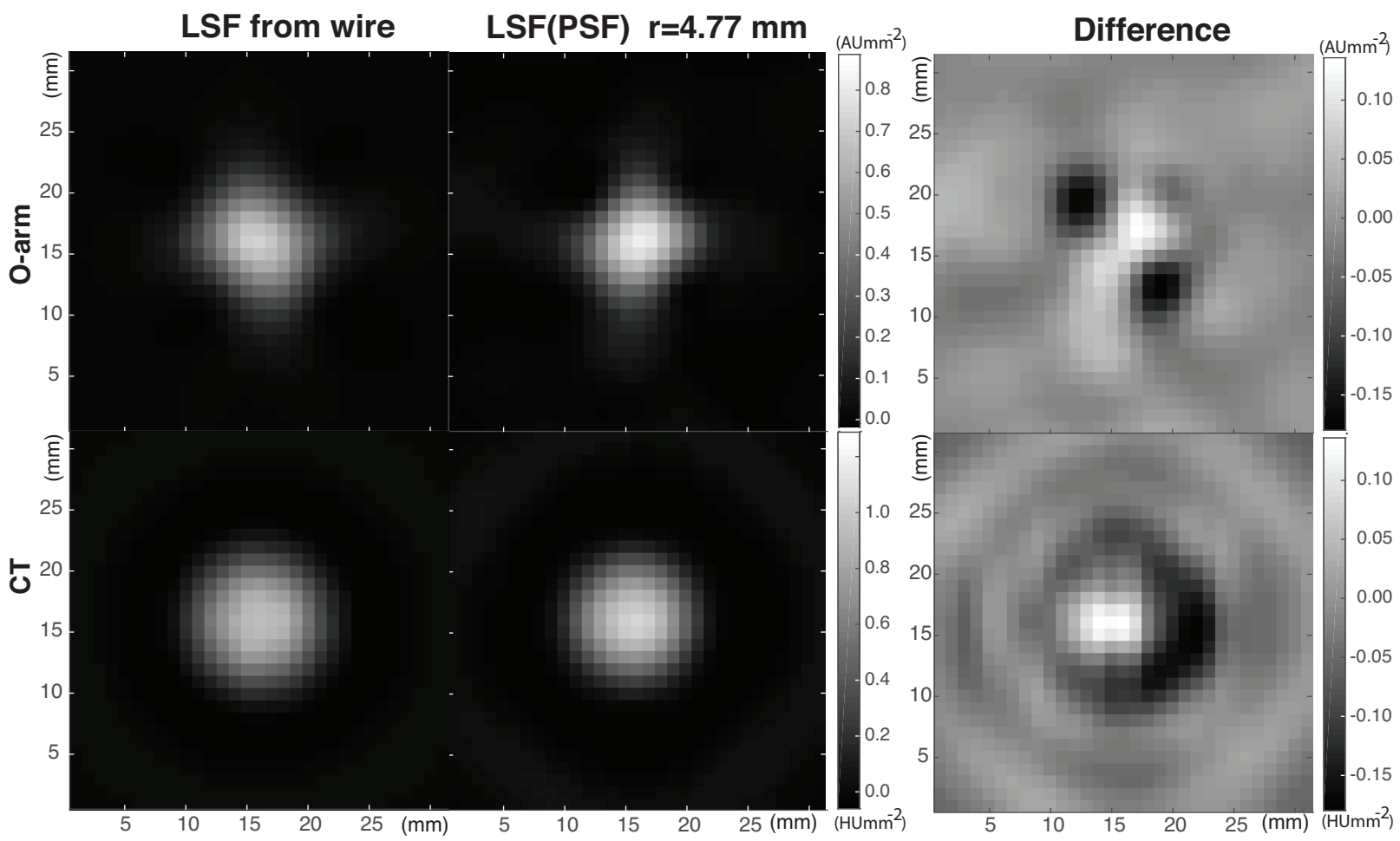

FIG. 8. Line spread functions shown as images. The LSF estimated from a wire segment and the LSF estimated from a $4.77 \mathrm{~mm}$ radius sphere are shown as well as the difference between the 2 estimates.

\begin{tabular}{|c|c|c|c|c|}
\hline \multirow{2}{*}{$\frac{\text { Scanner }}{\text { O-arm }}$} & \multirow{2}{*}{$\frac{\text { Radius }(\mathrm{mm})}{4.77}$} & \multicolumn{3}{|c|}{ PSF COM Std Dev (voxel/mm) } \\
\hline & & $(0.055 / 0.046$ & 0.088/0.037, & $0.066 / 0.027)$ \\
\hline $\mathrm{O}-\mathrm{arm}^{c}$ & 9.52 & $(0.034 / 0.028$ & $0.072 / 0.030$ & $0.055 / 0.023)$ \\
\hline $\mathrm{O}-\operatorname{arm}^{c}$ & 19.05 & $(0.037 / 0.031$ & $0.072 / 0.030$ & $0.048 / 0.020)$ \\
\hline O-arm & 4.77 & $(0.057 / 0.047$ & 0.090/0.037, & $0.065 / 0.027)$ \\
\hline O-arm & 9.52 & $(0.037 / 0.031$ & 0.079/0.033, & $0.054 / 0.022)$ \\
\hline O-arm & 19.05 & $(0.044 / 0.037$ & $0.084 / 0.035$ & $0.051 / 0.021)$ \\
\hline $\mathrm{CT}^{c}$ & 4.77 & $(0.093 / 0.028$ & 0.095/0.040, & $0.068 / 0.029)$ \\
\hline $\mathrm{CT}^{c}$ & 9.52 & $(0.013 / 0.004$ & $0.151 / 0.064$ & $0.175 / 0.073)$ \\
\hline $\mathrm{CT}^{c}$ & 19.05 & $(0.035 / 0.010$ & $0.028 / 0.012$ & $0.038 / 0.016)$ \\
\hline $\mathrm{CT}$ & 4.77 & $(0.052 / 0.016$ & 0.039/0.017 & $0.087 / 0.037)$ \\
\hline $\mathrm{CT}$ & 9.52 & $(0.070 / 0.021$ & 0.018/0.008, & $0.085 / 0.036)$ \\
\hline $\mathrm{CT}$ & 19.05 & $(0.073 / 0.022$ & $0.045 / 0.019$ & $0.057 / 0.024)$ \\
\hline
\end{tabular}

TABLE III. Standard deviations of PSF center of mass obtained along the three principal image axes with sphere motion correction indicated via the superscript ${ }^{c}$ for 23 image from the O-arm and 20 images from the CT. Results for 3 of 5 radii are shown. 